\title{
Actitudes de los docentes frente a los entornos virtuales de aprendizaje
}

\author{
Daniela Andrea Nicoli \\ daniandrea7@hotmail.com \\ Facultad de Humanidades y Ciencias de la Educación \\ Universidad Nacional de Pilar \\ Pilar-Paraguay
}

\section{RESUMEN}

Los entornos virtuales de aprendizajes ofrecen una alternativa educativa válida para las exigencias del mundo actual afianzando la formación continua. Esto requiere de docentes tutores capaces de guiar el proceso de enseñanza aprendizaje en el medio virtual haciendo un uso óptimo de las herramientas tecnológicas disponibles en beneficio de los objetivos educativos propuestos. Por tal motivo el presente trabajo pretende describir la aceptación y las competencias docentes en el uso de un aula virtual. Es una investigación descriptiva donde se medirán ciertos indicadores, basados en el modelo TAM (Modelo de Aceptación de la Tecnología, para conocer las actitudes de los docentes que se desempeñan en la modalidad a distancia frente a los a través enfoques positivistas, de entrevistas y análisis estadísticos del aula y pos-positivistas, a través del software ATLAS. Ti 9.

A partir de los resultados obtenidos se pretende plantear lineamientos de acción para optimizar el uso de las herramientas que nos ofrecen los EVA (Entornos Virtuales de Aprendizaje) y mejorar el modelo educativo (virtual)

Palabras claves: entornos virtuales; modelo TAM; aceptación y competencia docente; modalidad a distancia. 


\title{
Teachers' attitudes towards virtual environments Learning
}

\begin{abstract}
E-learning environments offer a valid educational alternative for the demands of today's world, strengthening continuous training. This requires tutors capable of guiding the teaching-learning process in the virtual environment making optimal use of the technological tools available for the benefit of the proposed educational objectives. For this reason, this work aims to describe the acceptance and teaching skills in the use of a virtual classroom. It is a descriptive research where certain indicators, based on the TAM model, will be measured to know the attitudes of teachers who work in the distance modality in front of the VAS, through positivist approaches, surveys, interviews and analyzes statistics of the virtual classroom and post-positivists, through the software ATLAS.ti 9.

Based on the results obtained, it is intended to propose action guidelines to optimize the use of the tools offered by the EVA and improve the (virtual) educational model.
\end{abstract}

Keywords: virtual environments; TAM model; teaching acceptance and competence; remote mode

Artículo recibido: 02 noviembre. 2021 Aceptado para publicación: 28 noviembre 2021 Correspondencia: daniandrea7@ hotmail.com Conflictos de Interés: Ninguna que declarar 


\section{INTRODUCCIÓN}

En la sociedad de la información y el conocimiento, contamos con nuevos escenarios que permiten una mayor posibilidad de acceso a la información y facilitan la comunicación. Actualmente, con la difusión masiva y generalizada de la información, la posibilidad de “aprender online" está creciendo, y las instituciones educativas tienen el compromiso y/o responsabilidad de ofrecer alternativas que permitan canalizar las ventajas de estos nuevos escenarios para consolidar la profesionalización y la formación continua. En este contexto, la Educación a Distancia (E a D) está avanzando a pasos agigantados gracias a la posibilidad que nos brindan las TIC.

Lorenzo García Aretio define a la Educación a Distancia como: ${ }^{1}$

"Sistema tecnológico de comunicación bidireccional, que puede ser masivo y sustituye la interacción personal en el aula de profesor y alumno como medio preferente de enseñanza por la acción sistemática y conjunta de diversos recursos didácticos y el apoyo de una organización y tutorías que propician el aprendizaje independiente y flexible de los estudiantes"

Considerando este concepto, se puede enmarcar a la educación virtual dentro de la Educación a Distancia. La educación virtual es una modalidad educativa que facilita el manejo de la información, la interacción entre compañeros y docentes en forma sincrónica o asincrónica a través del ciberespacio, superando las barreras geográficas y temporales y favoreciendo de esta manera la formación continua mediante el aprendizaje autónomo. En esta modalidad, se cuenta con los medios necesarios para poder adquirir los conocimientos a través de internet y la disponibilidad de equipos portátiles o móviles (laptops, tabletas, teléfonos celulares portátiles, etc.).

El aprendizaje autónomo es posible y se afianza más aun a través de los medios de información y comunicación (TIC). El punto de partida del aprendizaje autónomo es la responsabilidad y control personal del proceso de aprendizaje, avanzando al propio ritmo. Si a ese aprendizaje le damos un marco formal, podemos hablar de educación virtual, donde el docente que enseña tiene un papel activo, de ayuda y orientación, pero no es el protagonista, rol que asume el estudiante.

\footnotetext{
${ }^{1}$ GARCIA ARETIO, Lorenzo. (1987) "Hacia una definición de Educación a Distancia". Boletín informativo de la Asociación Iberoamericana de Educación superior de Educación a Distancia. Abril. Año 4. No 18. 4pp.
} 
Cuando hablamos de aulas virtuales hacemos referencias a entornos donde están presentes todos los elementos de un aula tradicional: alumnos, profesores, información, interacción, procesos evaluativos, etc. Este entorno educativo se caracteriza fundamentalmente por la separación física y temporal de los alumnos / profesores y porque el alumno realiza las actividades en forma independiente, organizando su propio tiempo y espacio; el docente tiene que asumir el liderazgo, ya no como poseedor absoluto del conocimiento sino como estratega para plantear métodos que guíen la búsqueda de la información, métodos que permitan el trabajo colaborativo, y fomentar a su vez la responsabilidad que implica aprender en este nuevos escenarios de aprendizaje.

\section{La utilización de un entorno virtual no es la solución a los miles de problemas} educativos de la actualidad, pero cabe destacar las ventajas que nos brindan:

- Permiten presentar la información utilizando diferentes medios, por ejemplo, textos, gráficos, animaciones, videos, etc.

- Favorecen el desarrollo del pensamiento crítico, a la vez que promueven el aprendizaje autónomo del alumno, con un rol más activo y responsable.

- Ofrecen condiciones favorables para el aprendizaje cooperativo, permitiendo comprender y compartir experiencias en relación con un determinado tema, de esta manera se potencia y socializa el aprendizaje.

- Colocan al docente en un rol de facilitador y guía del aprendizaje, a través de modelos pedagógicos más flexibles y constructivos.

- Brindan la posibilidad de acceder al aula desde cualquier lugar y punto geográfico, siempre que se cuente con los medios tecnológicos necesarios. (Cabero, 2004):

\section{Esta realidad presenta también nuevos desafíos, entre ellos:}

- La posibilidad igualitaria de acceso a esta nueva opción educativa mediada por las Tics: la educación virtual.

- Contar con profesionales en el área, que sean capaces de afianzar las ventajas que un aula virtual posee y de encontrar estrategias válidas para superar o minimizar los inconvenientes o desventajas.

Potenciar el uso del entorno virtual maximizando los recursos humanos y tecnológicos con que cuenta la institución es un desafío que hay que enfrentar; conocer las causas del escaso uso de las herramientas que posee un entorno virtual es el primer paso ante el 
desafío mencionado. Superado este primer paso, las instituciones, en este caso la U.N.P. ${ }^{2}$ (Universidad Nacional de Pilar), ampliarán sus horizontes a nivel regional, nacional e internacional, brindando la oportunidad de una educación continua y permanente para todas aquellas personas que, por motivos, laborales, familiares, geográficos y económicos no pueden acceder diariamente a un centro de estudios.

El tener una visión de la aceptación de los docentes y competencias de los mismos en esta área permitirá plantear planes de acción permitentes con miras a lograr el desafío antes mencionado.

Teniendo en cuenta todo lo que implica el aprendizaje mediado por TIC, el presente trabajo pretende indagar la aceptación y las competencias docentes en el uso de un aula virtual.

Se realizó una revisión de bibliografía sobre el tema en estudio, incluyendo una breve reseña histórica sobre la modalidad a distancia, el modelo e-learning y b-Learning, las diferentes plataformas educativas con las herramientas que cada una de ellas nos brindan y las competencias necesarias para que los tutores virtuales tengan un desempeño óptimo. La aceptación de los docentes frente a los entornos virtuales será analizada teniendo en cuenta el modelo TAM, modelo de Aceptación de Tecnológica (Davis y otros 1989), creado para explicar el uso de las tics en diferentes ambientes y comprobar cómo los usuarios aceptan la tecnología, considerando sus actitudes e intenciones, basándose en la Teoría de Acción Razonada (Ajzen y Fishbein, 1980); esta teoría pretende predecir la conducta de las personas considerando actitudes, intenciones, presión social, convicciones y conductas. TAM establece cuatro variables que determinan el uso efectivo de la tecnología:

- Facilidad percibida: "grado en el cual una persona cree que utilizando un sistema particular se liberará del esfuerzo"

- Utilidad percibida: definido por Fred Davis como "grado en el cual una persona cree que utilizando un sistema en particular mejorará su rendimiento en el trabajo".

- Actitud hacia su uso: conjunto de creencias y cogniciones en general dotadas de ciertas cargas afectivas, que predisponen para la acción ante determinas situaciones.

\footnotetext{
${ }^{2}$ La Universidad Nacional de Pilar, particularmente la Facultad de Derecho, Ciencias Políticas y Sociales, es la primera institución pública en brindar carreras de grado a distancia, aprobadas por CONES (Consejo Nacional de Educación Superior)
} 
- Intención de uso: grado en el que una persona ha formulado planes conscientes para desarrollar o no alguna conducta futura

Finalmente, se hará un análisis de los resultados obtenidos a través de una encuesta y entrevista en profundidad a los docentes involucrados en la modalidad a distancia de la carrera de la Derecho de la Universidad Nacional de Pilar.

\section{ESTRATEGIAS METODOLÓGICAS}

\section{Descripción del lugar de estudio}

El presente estudio se centrará en los docentes de la Carrera de Derecho de la Universidad Nacional de Pilar en la modalidad a distancia, modalidad habilitada desde el año 2009, a través del sistema tradicional, mediante la utilización de materiales impresos. Desde el año 2014 se ha implementado como apoyo la utilización del aula virtual Claroline.

\section{Tipo y método de estudio}

El trabajo de investigación será de carácter descriptivo, se medirán una serie de indicadores que permitirán especificar la aceptación y competencias de los docentes en el uso de un aula virtual.

Como establece R. H. Sampieri (2004) "Desde el punto de vista científico describir es medir. Esto es, en un estudio descriptivo se selecciona una serie de cuestiones y se mide o recolecta información sobre cada una de ellas, para sí -valga la redundancia- describir lo que se investiga." (p.118)

El estudio será ex post facto, transversal y no experimental.

El método de investigación será cuanti- cualitativo.

Para la parte cuantitativa se tomará el enfoque positivista, basándose en el análisis porcentual de las respuestas obtenidas en las encuestas, datos estadísticos disponibles en el aula virtual y análisis de las entrevistas realizadas a informantes claves.

Para realizar la parte cualitativa, se tomarán el paradigma Pos-positivistas, con enfoque inductivo, a través de los informantes o fuentes, me permite conocer la realidad propia de un determinado sector de la sociedad, comunidad o informantes, procesando y contrastando información, creando teorías en función de la información extraída. El investigador es el propio instrumento de recolección de datos.

A partir de una ruta investigativa, partiendo de una pregunta-problema, de donde surgen las categorías orientadoras del estudio, luego del relevamiento de datos, realizó un proceso de convergencia, a través de una codificación, luego se procedió a la parte 
analítica a través de un análisis de co- ocurrencia código-documento, redes y posteriormente la fase de interpretación con el cálculo de índice de emergencia, que determina los elementos resaltantes de la investigación.

\section{Fuentes de Datos}

- Datos estadísticos del aula virtual

- Bibliografía

- Documentos institucionales

- Encuestas.

- Entrevistas a informantes claves.

\section{Población}

El estudio tomó la totalidad de los docentes involucrados en la modalidad a distancia en la carrera de Derecho, de la Facultad de Derecho, Ciencias Políticas y Sociales de la U.N.P. Total de docentes en la carrera: 41

\section{Técnicas de recolección de datos.}

\subsection{Fase cuantitativa:}

Encuestas: A los docentes involucrados en la modalidad a distancia

Datos estadísticos del aula virtual

\subsection{Fase Cualitativa.}

- Se planearon las preguntas de investigación a través de la observación de trabajo docente en el aula virtual.

- Selección de los informantes claves, de acuerdo a criterios de inclusión.

- Aplicación de la pregunta de investigación.

\section{Análisis de datos}

Para el análisis de datos cuantitativos, se tabularon y analizaron estadísticamente (estadística descriptiva) mediante cuadros de porcentajes interpretados según los indicadores previstos.

El análisis cualitativo se realizó a través de la utilización de categorías conceptuales, presentando los resultados en tablas y gráficos.

\section{En la fase cualitativa se realizaron los siguientes pasos:}

- Selección de los informantes claves, de acuerdo a criterios de inclusión.

- Aplicación de la pregunta de investigación. 
- Las respuestas se convierten en las categorías orientadoras (CO), en función de la emergencia (pregunta de investigación.)

- De estas CO, se derivan otras preguntas, más específicas, amplia el espectro de información respecto a las respuestas de los informantes claves.

- Abrir la respuesta del informante1 y empezar a codificar

- Los grupos de códigos van a surgir como una categoría fundamentada, surgen directamente de la investigación, en función del grado de Densidad y de Enraizamiento

- Realización la tabla de enraizamiento y densidad.

- Enraizamiento: se refiere a la relación de las citas dentro de los códigos, es decir, representa el número de citas que hacen mención del código

- Densidad: tiene relación con la vinculación y el tipo de relación entre los códigos, es decir, representa el número de veces que un código se relaciona con otros.

- Realización una red semántica. Permiten la Vinculación grupo-código: hace alusión a la vinculación de los códigos con los grupos de códigos.

- Cálculo de los códigos de emergencias.

Para el procesamiento de datos se dividieron aleatoriamente en dos grupos y así comparar los mismos reforzando la confiabilidad del instrumento.

La validación de los instrumentos utilizados en el estudio cualitativo está dada por la credibilidad y la confortabilidad.

\section{La primera basada en:}

- Permanencia en el contexto estudiado permitiendo la apropiación de la realidad estudiada

- La observación persistente de forma amplia, sistemática y detallada a fin de brindar profundidad al estudio

- Triangulación de los datos obtenidos de diferentes fuentes y a través de diversas técnicas.

La segunda como garantía de que los descubrimientos no están sesgados por la subjetividad del investigador, según Yuni y Urbano (2005), discutiendo los resultados con los informantes, realizando procesos de triangulación de los datos y explicando la posición del investigador, este parte de la premisa que el contex to es relativo y solo puede 
ser entendido desde el punto de vista de los actores estudiados. La realidad no cambia por las observaciones y mediciones realizadas.

\section{RESULTADOS Y DISCUSIÓN.}

\subsection{Fase Cuantitativa.}

Las primeras preguntas de la encuesta que apuntan a la aceptación de los docentes de los entornos virtuales, se evidencia un porcentaje alto (más del $50 \%$ ) que responden estar totalmente de acuerdo con la credibilidad del entorno virtual, como así también a la efectividad del mismo, tanto para la formación de los alumnos como para la labor docente, sintiéndose a gusto trabajando en el ámbito virtual el $85 \%$ de los docentes consultados. Si bien el $67 \%$ responde que la capacitación recibida en el manejo del aula virtual ha sido suficiente, también un alto porcentaje expresa que la ayuda técnica es necesaria, por lo que podemos deducir que aún no poseen el manejo cabal del entorno virtual, y las herramientas que este ofrece. Es así que solo el 41, 1 \% utiliza mensajería o participación en foros para comunicarse con los alumnos, y un alto porcentaje $47 \%$ lo realiza a través de WhatsApp y otros medios, como llamadas telefónicas.

El tiempo de dedicación al aula virtual, que corresponde al porcentaje más elevado, expresa que ingresa al aula una vez por semana $(41,2 \%)$ y cuando los plazos académicos y administrativos lo requieren, en los meses de agosto, septiembre y octubre en el primer semestre y en los meses de febrero, marzo y abril en el segundo semestre, $(38,2 \%)$ con una dedicación de 2 a 4 horas semanales.

Un factor que dificulta la labor docente es la conectividad, cuyo mayor porcentaje establece que es regular (44\%), solo el $8.8 \%$ especifica que es excelente.

Las preguntas relacionadas con las habilidades en el manejo de las herramientas en la encuesta realizada, los porcentajes más elevados dan una repuesta positiva a los siguientes planteamientos:

a. Da la bienvenida a los estudiantes que participan en el curso en red a través de foros de presentación o mensajes internos

b. Da información, clarifica y explica los contenidos presentados.

c. Responde a los trabajos de los estudiantes, permitiendo retroalimentación

d. Propone foros de discusión

e. Realiza actividades formativas específicas a través de cuestionarios o tareas

f. Gestiona los grupos de aprendizaje que formen para el trabajo en la red. 
g. Incorpora y modifica nuevos materiales al entorno formativo

h. Propone actividades para facilitar el conocimiento (personal y académico) entre los participantes

i. Establece el acceso a los contenidos de los diferentes temas en base a criterios como la temporalización o la fecha de entrega de actividades correspondientes a cada uno de los módulos

j. Planifica las sesiones de chat en lo referente a horarios, temáticas, etc.

Sin embargo, al ingresar al aula virtual se ha evidenciado que las actividades realizadas por los docentes en la misma se pueden sintetizar en las siguientes:

- Descarga y corrección de trabajos. (colocando puntajes logrados por el alumno y sugerencias de mejoras y/o retroalimentación)

- Descarga de documentos informativos

- Adjuntar imágenes

- Agregar anuncio (Sobre plazos, fechas de entregas de trabajos)

- Adjuntar documentos con información complementaria

- Adjuntar cuestionarios (Facilitando la autoevaluación de los alumnos)

- Adjuntar video (Explicativos de los temas desarrollados)

- Adjuntar actividades formativas y de repaso previo al periodo de exámenes.

No se evidencio el uso de foros, chat y mensajería interna del aula. Al mismo tiempo se evidencia que el ingreso de los docentes al aula virtual es más frecuente cuando se cierran los periodos evaluativos.

Si hacemos un paralelo entre lo expresado en las encuestas y lo evidenciado en aula virtual tenemos lo siguiente:

\begin{tabular}{lcc}
$\begin{array}{c}\text { Actividades desempeñadas por los } \\
\text { docentes }\end{array}$ & $\begin{array}{c}\text { Repuesta de Encuesta } \\
\text { (con mayor porcentaje) }\end{array}$ & $\begin{array}{c}\text { Análisis de datos del aula } \\
\text { virtual }\end{array}$ \\
$\begin{array}{l}\text { Da la bienvenida a los estudiantes } \\
\text { que participan en el curso en red a } \\
\text { través de foro de presentación o } \\
\text { mensajes internos }\end{array}$ & $\mathrm{Si}$ & $\mathrm{No}$ \\
\hline $\begin{array}{l}\text { Da información, clarifica y explica } \\
\text { los contenidos presentados. }\end{array}$ & $\mathrm{Si}$ & $\mathrm{Si}$ \\
\hline $\begin{array}{l}\text { Responde a los trabajos de los } \\
\text { estudiantes, permitiendo } \\
\text { retroalimentación }\end{array}$ & $\mathrm{Si}$ & $\mathrm{Si}$ \\
\hline
\end{tabular}




\begin{tabular}{|c|c|c|}
\hline $\begin{array}{c}\text { Actividades desempeñadas por los } \\
\text { docentes }\end{array}$ & $\begin{array}{l}\text { Repuesta de Encuesta } \\
\text { (con mayor porcentaje) }\end{array}$ & $\begin{array}{c}\text { Análisis de datos del aula } \\
\text { virtual }\end{array}$ \\
\hline Propone foros de discusión & $\mathrm{Si}$ & No \\
\hline $\begin{array}{l}\text { Realiza actividades formativas } \\
\text { específicas a través de } \\
\text { cuestionarios o tareas }\end{array}$ & $\mathrm{Si}$ & $\mathrm{Si}$ \\
\hline $\begin{array}{l}\text { Gestiona los grupos de aprendizaje } \\
\text { que formen para el trabajo en la red. }\end{array}$ & $\mathrm{Si}$ & No \\
\hline $\begin{array}{l}\text { Incorpora y modifica nuevos } \\
\text { materiales al entorno formativo }\end{array}$ & $\mathrm{Si}$ & $\mathrm{Si}$ \\
\hline $\begin{array}{l}\text { Propone actividades para facilitar } \\
\text { el conocimiento (personal y } \\
\text { académico) entre los participantes }\end{array}$ & $\mathrm{Si}$ & No \\
\hline $\begin{array}{l}\text { Establece el acceso a los contenidos } \\
\text { de los diferentes temas en base a } \\
\text { criterios como la temporalización o } \\
\text { la fecha de entrega de actividades } \\
\text { correspondientes a cada uno de los } \\
\text { módulos }\end{array}$ & $\mathrm{Si}$ & $\mathrm{Si}$ \\
\hline $\begin{array}{l}\text { Planifica las sesiones de chat en lo } \\
\text { referente a horarios, temáticas, etc. }\end{array}$ & $\mathrm{Si}$ & No \\
\hline
\end{tabular}

Tabla 1 Actividades que desempeñan los docentes. Fuente: Elaboración propia.

Estas discrepancias en las respuestas fueron aclaradas en las entrevistas donde los docentes manifestaron que realizan las actividades mencionadas, pero a través de otros medios tecnológicos, generalmente el telefónico.

Las horas de dedicación son entre 2 horas a 4 horas que son las fijadas por la institución para tutorías.

\section{Fase Cualitativa.}

Las preguntas relacionadas con la aceptación de los docentes de los EVA, en base a los códigos de las respuestas tenemos un mayor enraizamiento de los aspectos positivos resaltándose la posibilidad de acceso a los medios múltiples, como ser uso de link, foros y archivos; el entorno agradable de trabajo, que facilitan el trabajo docente y la autonomía de aprendizaje de los alumnos. El aspecto negativo evidenciado es la escasa conectividad. En este sentido la Categoría R 1 (Ventajas y desventajas de los EVA), està asociada con el acceso a los medios múltiples, la posibilidad de acceso a la información y el desarrollo de destrezas y habilidades tecnológicas e intelectuales. 
Figura 1 Códigos emergentes

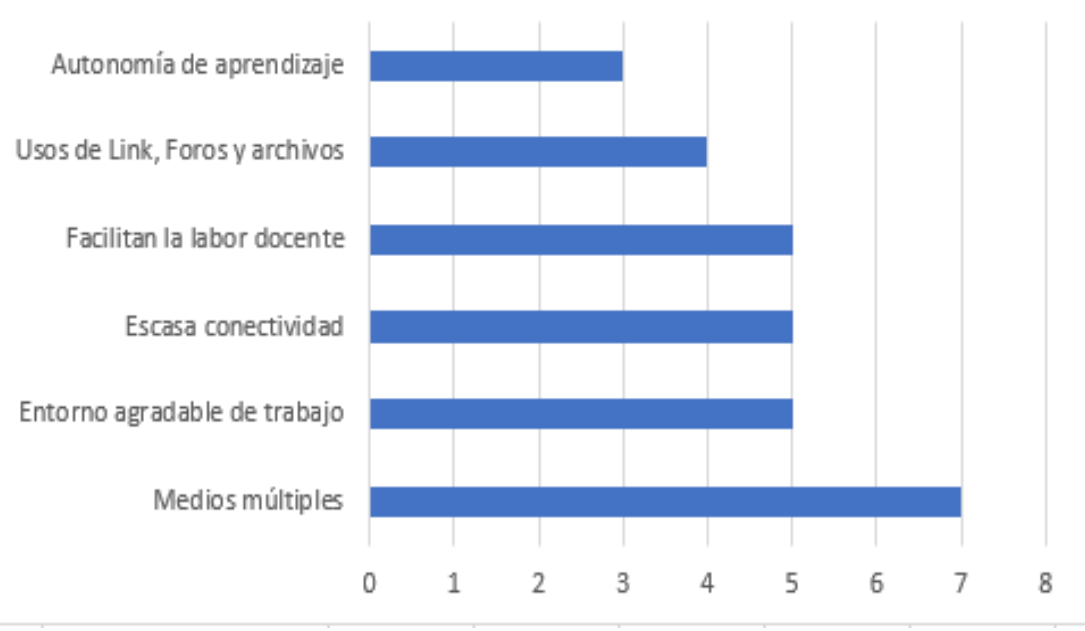

Fuente: Elaboración propia

Al mismo tiempo la escasa conectividad está asociada a la dificultad de la participación sincrónica de los estudiantes.

La categoría R 2 se evidencia una relación entre el entorno agradable de trabajo y la posibilidad de facilitar la labor docente esto asociado al acceso a la información a través de los múltiples medios, entre ellos la grabación de clases, lo que facilita el desarrollo de ciertas habilidades y destrezas.

Figura 2 Asociaciones de códigos a la Pregunta orientadora: Ventajas y Desventajas (R1)

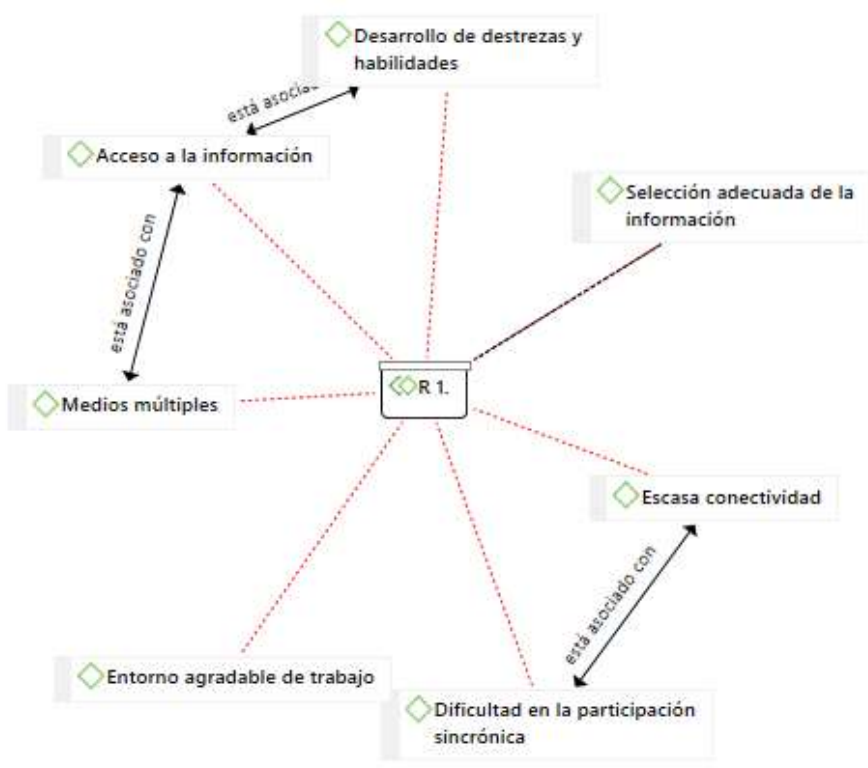

Fuente: Elaboración propia 
La categoría R 3 se resalta la posibilidad de acceso a diversas herramientas tecnológicas asociada con la utilización de foros, link, archivos, videoconferencias y WhatsApp. Se visualiza nuevamente asociada con esta categoría la dificultad de participación sincrónica por la escasa conectividad, y la falta de manejo de algunas herramientas que ofrecen los tics.

Figura 3 Asociaciones de códigos a la pregunta orientadora Herramientas más utilizadas y menos utilizadas (R3)

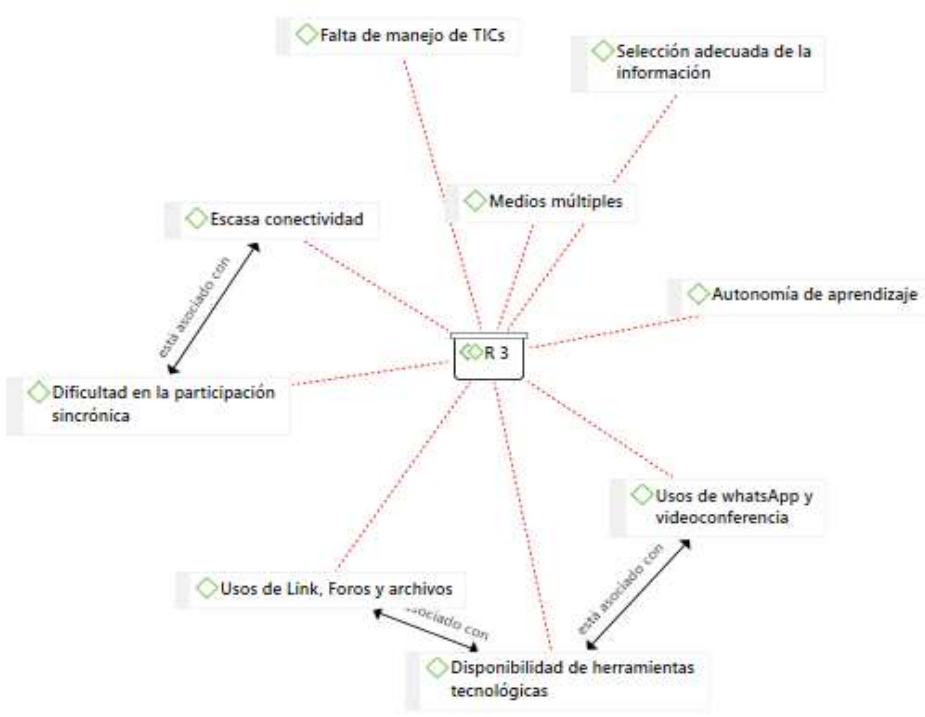

Fuente: Elaboración propia

Como tutor manifiestan que, aún necesitan más seguridad y practica en el manejo de las herramientas que el entorno virtual ofrece, de allí la importancia del apoyo del equipo técnico. Algunos manifestaron interés en tener más talleres del área.

La categoría R 4, relacionada con la actitud docente hacia los EVA, se visualización dos aspectos claves y relacionados: Facilitan la labor docente y el entorno agradable de trabajo.

Figura 4 Asociaciones de códigos a la pregunta orientadora Herramientas más utilizadas y menos utilizadas (R4)

Fuente: elaboración propia

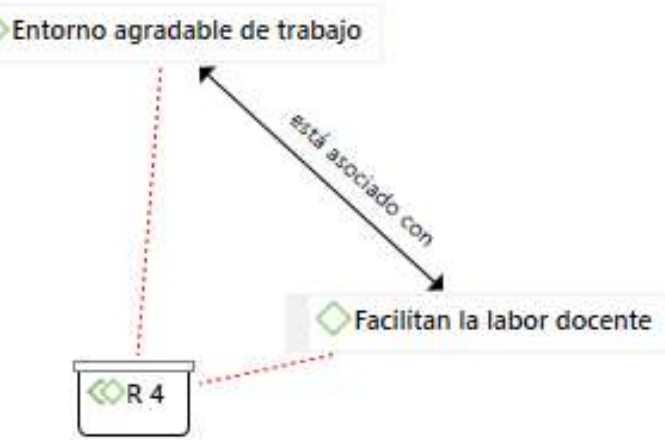


Los docentes entrevistados poseen equipos y conexión a internet, algunos argumentaron la necesidad de contar en la institución de un espacio "bien equipado" exclusivo para uso de los docentes de modalidad a distancia. El término bien equipado hace referencia a computadoras, conexión a internet en forma estable, espacio para filmaciones de videos tutoriales, etc.

A partir de los hallazgos obtenidos, se puede determinar que los docentes evidencian una aceptación de los EVA. Los resultados guardan relación con los parámetros establecidos a partir del modelo TAM que determinan la aceptación y uso efectivo de la tecnología; la utilidad, la actitud, intención y la facilidad percibida, relacionando está última sobre la posibilidad de acceso a los medios múltiples (recursos para brindar información, según lo evidenciado en aula virtual).

La definición de Aretio de Educación a Distancia, dentro de la cual podemos enmarcar la educación virtual, se establece claramente la importancia de las tutorías para el aprendizaje flexible y autónomo de los estudiantes, la interacción en forma sincrónica o asincrónica, sin embargo, en los resultados obtenidos se evidencia la escasa o nula utilización de herramientas que poseen los EVA para la interacción entre docentes y alumnos. En este aspecto el parámetro facilidad, establecido por el modelo no aplica, resulta más fácil la utilización de otros medios tecnológicos.

Si consideramos las funciones que desempeñan los tutores en el aula se evidencian en cuanto a la función pedagógica la optimización del proceso de enseñanza aprendizaje mediante la facilitación de recursos y actividades de acuerdo a los contenidos y objetivos de la materia, cumpliendo lo establecido por el CO.N.E.S (Consejo Nacional de Educación Superior ) en el Reglamento de Educación a Distancia en su artículo 17 y artículo 19 en este sentido; sin embargo las "habilidades comunicativas adecuadas a este entorno" y "feedback", también mencionadas en dichos artículos, los resultados son escasos o nulos. Los docentes se valen de otros medios tecnológicos.

\section{CONCLUSIONES Y RECOMENDACIONES}

\section{Conclusiones}

Considerando los resultados obtenidos, las variables establecidas por el modelo TAM, y los objetivos de la investigación se establecen las siguientes conclusiones, con el fin de elaborar lineamientos para la mejora del modelo educativo a partir del conocimiento de 
las causas por la que los docentes no utilizan en su total potencial las herramientas que brinda el entorno virtual.

La aceptación de las docentes del entorno virtual, teniendo en cuenta el modelo antes mencionado, está basada en la utilidad, la actitud y la intención. Esto se evidencia en la aprobación en cuanto a que facilita el trabajo docente, el avance en el uso del entorno virtual desde el comienzo de la modalidad a distancia hasta la actualidad y su predisposición para seguir aprendiendo.

Si consideramos la variable utilidad consideran el uso del entorno virtual como algo destacado en su labor docente y que mejorará su rendimiento.

En cuanto a su actitud hay un sentimiento positivo para utilizar los EVA, afirman su credibilidad y efectividad en el proceso enseñanza-aprendizaje, considerándolo en entorno agradable de trabajo.

En cuanto a la intención, los docentes han participado de las capacitaciones por lo que evidencia los planes y la intención de usar los EVA, esta intención se afianza en algunos docentes que han expresado sus deseos de continuar con capacitaciones que le permitan entender mejor los aspectos operativos de los EVA

Sin embargo, se pudo detectar debilidades en cuanto a las habilidades técnicas en el manejo de las herramientas que el entorno virtual ofrece, los docentes necesitan afianzarse en este aspecto y así optimizar su uso en el proceso de enseñanza aprendizaje. Sin bien manifiestan que facilita la labor docente, entre otras cosas por los múltiples medios que ofrece, también manifiestan un escaso manejo en las herramientas que los EVA brindan, fundamentalmente aquellas que permiten la comunicación, como ser mensajería interna, chat, foros, etc., por lo que optan por otros medios tecnológicos.

Los docentes poseen habilidades pedagógicas que se evidencia a través del trabajo realizado través del ámbito virtual planteando tareas evaluativas, corrigiendo, sugiriendo mejoras, agregando anuncios de interés, etc. y habilidades comunicativas que se evidencia a través de la utilización de otros medios tecnológicos como ser teléfono y/o correos electrónicos, para responder a las inquietudes de los alumnos, aclarar dudas, etc. Empero necesitan afianzar sus habilidades técnicas para optimizar el uso del entorno virtual, y que este deje de ser un repositorio de información para pasar hacer un medio más atractivo, amigable, interactivo y dinámico utilizando las herramientas de comunicación que ofrece el mismo. 
Otro factor que dificulta la labor docente es conectividad regular a internet, en forma particular. Institucionalmente esa conectividad ha mejorado mediante labor técnica en la redistribución de la red, contando con una línea exclusiva para la modalidad a distancia.

\section{Recomendaciones}

Se recomienda ofrecer capacitaciones /talleres donde se fomenten y/o afiancen las habilidades técnicas de los docentes en cuanto a el dominio de las herramientas que ofrecen los EVA, fundamentalmente las diferentes las herramientas de comunicación que le permitan mantener una comunicación efectiva, entre tutor -alumno y entre los alumnos, y así realizar una labor tutorial personalizada y motivadora a través de los EVA.

El planteamiento de talleres en forma continua permitirá crear espacios de prácticas en el uso y manejo de los EVA, y un ámbito aclarar dudas y compartir experiencias de la labor como tutor.

\section{Estos talleres deben tener como objetivos fundamentales que los tutores logren:}

- Utilizar efectivamente las herramientas de comunicación que ofrecen los EVA

- Crear y generar entornos de aprendizajes colaborativos

- Afianzar la compresión en los aspectos operativos de los EVA.

Al mismo tiempo se sugiere contar con un espacio confortable y equipado exclusivo para los tutores de la modalidad a distancia que les permita realizar su labor sin interrupciones y crear un espacio de trabajo colaborativo: "un recinto de producción y aprendizaje"

\section{LISTA DE REFERENCIAS}

Área Moreira, Manuel (2006). La educación a distancia. De la teoría a la práctica, Barcelona, Editorial Ariel.

Cabero Almenara, J. (2006). Bases pedagógicas del e-learning. RUSC. Universities and Knowledge Society Journal, 3 (1), 0. Universitat Oberta de Catalunya Barcelona, España. Http

Cabero, J. y Barroso, J., (2012). El tutor virtual: Características y funciones. En Padilla G., Leal, F., Hernández, M. y Cabero, J. (Eds.), Un reto para el profesor del futuro: La tutoría virtual (pp. 15- 42). Printed by Publidisa: SINED.

Cabero, J. y Llorente, M.C. (2005). Las plataformas virtuales en el ámbito de la tele formación, en Revista electrónica Alternativas de Educación y Comunicación. [Archivo

$\mathrm{PDF}]$ 
http://tecnologiaedu.us.es/cuestionario/bibliovir/plataformas_virtuales_tel eformacion 2005.pdf

Cabero, J. y Román, P. (2006). E-actividades. Un referente básico para la formación en Internet. Sevilla: MAD, S. L.

Castaño Garrodo, C. (2003) "El rol del profesor en la transición de la enseñanza presencial al aprendizaje En Rev comunicar, núm 21, «on line Huelva. España. http://www.redalyc.org/pdf/158/15802107.pdf

Ferrante, Adela (2000). "Educación a distancia, virtualidad y cambios en la concepción del espacio", en El habitar urbano: pensamiento, imaginación y límite, Buenos Aires, Ciudad Argentina-Usal.

García Areito, Lorenzo. Módulo III “El tutor y la enseñanza a distancia ”. Universidad Nacional de Educación a Distancia . pág. 115

García Aretio, L. (2006). La Educación a Distancia. De la teoría a la práctica, Barcelona, Ariel Educación.

García Aretio, Lorenzo. Historia de la educación a distancia. RIED. Revista Iberoamericana de Educación a Distancia, [S.1.], v. 2, n. 1, p. 8-27, ene. 1999. ISSN 1390-3306.

<http://revistas.uned.es/index.php/ried/article/view/2084/1959>. Fecha de acceso: 07 nov. 2019 doi: https://doi.org/10.5944/ried.2.1.2084

García Aretio, Lorenzo. (1987)“Hacia una definición de Educación a Distancia”. Boletín informativo de la Asociación Iberoamericana de Educación superior de Educación a Distancia. Abril. Año. $N^{o} 18.4 p p$.

Hidalgo, F. Ventajas y desventajas del Software libre y comercial. (2011) www.uclflormaria.blogspot.com/2011/03/ventajas-y-desventajas-delsoftware.html Fecha: 5/07/18

Llorente Cejudo, $\mathrm{M}^{\mathrm{a}}$ del Carmen (2007). El tutor en E-learning: aspectos a tener en cuenta. Revista Electrónica de Tecnología Educativa (Edute). Núm.23/ Julio 2007.

López, M. (2007). ¿Es indispensable repensar la mediación pedagógica del tutor de la educación a distancia actual? Revista ipac. ISSN 1993-6850. RNPS No. 2140. http://www..iplac.rimed.cu/index.php?option=com_content\&view=article\&id=1 81:mediaciedagadel-tutor-en-ead \&catid=21\&Itemid=118 
Maldonado , Daniel Martin (2018), http://empresayeconomia.republica.com/recursoshumanos/claroline-una-plataforma-educativa-virtual.html

Nieto , H., De Majo O. "Historia de la Educación a Distancia en Argentina”

Palacios, R. (2007). La tutoría: Una perspectiva desde la comunicación y la educación. En Landeta, A. (Ed.), Buenas prácticas de e-learning (pp. 91-110). España: ANCED.

Plataformas de Software libre (o de investigación y colaboración). https://www.sites.google.com/site/plataformaseducativasvirtuales/home/tipos/so $\underline{\text { ftwarelibre }}$

Tendencias epistemológicas de la investigación científica en el Siglo XXI

Dr. José Padrón (josepadrong@fundacionlineai.org) Universidad Simón Rodríguez (Caracas, Venezuela). https://www.moebio.uchile.cl/28/padron.html

Rodríguez-Hoyos, C; Calvo Salvador, A. (2011) "La figura del tutor de e-learning. Aportaciones de una investigación con estudios de caso RUSC.”Rev Universities and Knowledge Society Journal, vol. 8, núm. 1. Universitat Oberta de Catalunya $\begin{array}{llll}\text { Barcelona, } & \text { España. } & \text { [Archivo }\end{array}$ http://www.redalyc.org/pdf/780/78017126004.pdf

Salinas-Ibáñez, J. (1). Entornos virtuales y formación flexible. Revista Tecnología En Marcha, 17(3), pág. 69-80. https://revistas.tec.ac.cr/index.php/tec_marcha/article/view/

Torres, S. y Ortega J.A. (2003). Indicadores de calidad en las plataformas de formación virtual: una aproximación sistemática. etic@ @et, $\mathrm{n}^{\circ} 1$. [Archivo PDF] http://www.ugr.es/ sevimeco/revistaeticanet/Numero1/Articulos/Calidade .pdf

Zapata Ros, M. (2003) Sistemas de gestión del aprendizaje - Plataformas de tele formación Learning Revista de Educación a Distancia núm. 50 Management Systems Universidad de Murcia. España. http://revistas.um.es/red/article/view/270821/198351 